\title{
Conflit(s) et public(s) : orientations bibliographiques
}

CHRISTIAN VEILLEUX

University of Toronto

1. Espace public et communautés conceptuelles

2. Antagonisme et culture

3. Publics confessionnels

4. Publics géo-/sociopolitiques et groupes linguistiques

5. Publics féminins

6. Monde du livre et lectorats

\section{Espace public et communautés conceptuelles}

Cette première section réunit principalement des travaux qui discutent des notions habermassiennes de "sphère publique structurée par la représentation " et de "sphère publique bourgeoise" (Habermas 2012 [1978]). Parmi les autres conceptualisations du public fréquemment rencontrées au fil des discussions, notons celles de "communauté interprétative " (Fish 1980), de "communauté imaginée " (Anderson 2016 [1983]), de " communauté textuelle » (Stock 1983) et de "communauté discursive " (Helgerson 1992). Cette dernière est mise à l'honneur par Yachnin et Eberhart (2015) dans la dernière publication du projet canadien Making Publics : Media, Markets, and Association in Early Modern Europe, 1500-1700, dirigé par Paul Yachnin (Université McGill, 2005-2010). Yachnin et Eberhart, dans un bref commentaire sur le choix terminologique de Helgerson, signalent une nuance d'ordre politique entre les idées de communauté et de public ${ }^{1}$.

\section{ANDERSON, Benedict, Imagined Communities : Reflections on the Origin and Spread of Nationalism (Londres : Verso, 2016 [1983]), 240 p.}

1. Yachnin et Eberhart, Forms of Association, p. 2 : «Discursive communities, like publics, are mobile and changeful, capable of overlapping one with another, and able to speak formatively about the whole nation from particular sectoral positions. However, [... c] ommunities tend by definition to be inwardlooking and closed to others; they are not bent on expansion, inclusiveness, and publicity [...] The very word "community" is sometimes used to name a kind of collective relationship bracketed off from the stress, struggle, and changeability of the political world ». 
BACKSCHEIDER, Paula R. et Timothy DYSTA L, éd., The Intersections of the Public and Private Spheres in Early Modern England (Londres, New York : Routledge, 2013 [1996]), 269 p.

BLAIR, Ann et Anja-Silvia GOEING, éd., For the Sake of Learning. Essays in Honor of Anthony Grafton, coll. «Scientific and Learned Cultures and Their Institutions » (Leyde, Boston : Brill, 2016), « Part II - Knowledge Communities », 105-211.

BLUME, Hermann, Christoph LEITGEB et Michael RÖSSNER, éd., Narrated Communities - Narrated Realities. Narration as Cognitive Processing and Cultural Practice (Leyde : Brill, 2015), 263 p.

CHIVALLON, Christine, «Retour sur la "communauté imaginée" d'Anderson. Essai de clarification théorique d'une notion restée floue ", Raisons politiques 27.3 (2007) : 131-172.

CROSSLEY, Nick et John Michael ROBERTS, éd., After Habermas. New Perspectives on the Public Sphere (Oxford : Blackwell, 2004), $184 \mathrm{p}$.

DOOLEY, Brendan, "News and Doubt in Early Modern Culture. Or, Are We Having a Public Sphere yet ? ", in The Politics of Information in Early Modern Europe, éd. Sabrina Alcorn Baron et Brendan Dooley, coll. « Routledge Studies in Cultural History " (Londres, New York : Routledge, 2001), 275-290.

EMDEN, Christian J. et David MIDGLEY, éd., Changing Perceptions of the Public Sphere (New York, Oxford : Berghahn Books, 2012), "Part I - Publics Before the Public Sphere », 15-73.

FISH, Stanley Eugene, Is There a Text in this Class? The Authority of Interpretive Communities (Cambridge : Harvard University Press, 1980), 394 p.

HABERMAS, Jürgen, L'Espace public : archéologie de la publicité comme dimension constitutive de la société bourgeoise, trad. Marc B. de Launay, coll. «Critique de la politique» (Paris : Payot, 2012 [1978]), 324 p.

HALASZ, Alexandra, The Marketplace of Print. Pamphlets and the Public Sphere in Early Modern England (Cambridge : Cambridge University Press, 1997), " The Public Sphere and the Market Place », 162-203.

HALVORSON, Michael et Karen E. SPIERLING, éd., Defining Community in Early Modern Europe, coll. "St. Andrews Studies in Reformation History» (Aldershot, Burlington : Ashgate, 2008), 364 p.

HELGERSON, Richard, Forms of Nationhood: The Elizabethan Writing of England (Chicago : University of Chicago Press, 1992), 367 p. 
JOUHAUD, Christian et Alain VIALA, éd., De la Publication entre Renaissance et Lumières (Paris : Fayard, 2002), $365 \mathrm{p}$.

KIRBY, Torrance, Persuasion and Conversion : Essays on Religion, Politics, and the Public Sphere in Early Modern England, coll. "Studies in the History of Christian Traditions » (Leyde, Boston : Brill, 2013), «Public Forum and Forum of the Conscience : John Calvin's Groundwork of the Modern Public Sphere », 36-50.

LAKE, Peter et Steven PINCUS, "Rethinking the Public Sphere in Early Modern England ", in The Politics of the Public Sphere in Early Modern England, éd. Peter Lake et Steven Pincus, coll. «Politics, Culture, and Society in Early Modern Britain " (Manchester : Manchester University Press, 2007), 1-30.

MCKEON, Michael, "What Was an Early Modern Public, and How Was It Made? », History Compass 10.9 (2012) : 714-730.

MEARS, Natalie, Queenship and Political Discourse, coll. «Cambridge Studies in Early Modern British History " (Cambridge : Cambridge University Press, 2005), « The Elizabethan Public Sphere », 182-216.

MERLIN-KAJMAN, Hélène, "Le public au XVII ${ }^{e}$ siècle et au-delà selon Auerbach ", in Erich Auerbach : la littérature en perspective, éd. Paolo Tortonese (Paris : Presses Sorbonne nouvelle, 2009), 91-116. , "Le public et ses envers, ou l'archaïsme de Furetière ", Littératures classiques 47 (2003) : 345-380.

, "Le public : quelques réflexions historiques ", in Les Sens du public, éd. Daniel Cefaï et Dominique Pasquier (Paris : Presses Universitaires de France, 2003), 101-112.

, Public et littérature en France au XVII e siècle (Paris : Les Belles Lettres, 1994), $477 \mathrm{p}$.

MICHON, Cédric, « Du bon usage de l'anachronisme en histoire : l'opinion publique à la Renaissance », in L'Opinion publique en Europe (1600-1800), éd. Lucien Bély (Paris : Presses de l'Université Paris-Sorbonne, 2011), $38-67$.

MUIR, Edward, " The Idea of Community in Renaissance Italy », Renaissance Quarterly 55.1 (2002) : 1-18.

PETTEGREE, Andrew, «A Provincial News Community in Sixteenth-Century Europe », in Public Opinion and Changing Identities in the Early Modern Netherlands : Essays in Honour of Alastair Duke, éd. Judith Pollmann et 
Andrew Spicer, coll. « Studies in Medieval and Reformation Traditions » (Leyde, Boston : Brill, 2007), 33-48.

RENDALL, Steven, «Reading in the French Renaissance. Textual Communities, Boredom, Privacy ", in Reading the Renaissance. Culture, Poetics, and Drama, éd. Jonathan Hart (New York, Londres : Garland, 1996), 35-44. ROSPOCHER, Massimo, éd., Beyond the Public Sphere : Opinions, Publics, Spaces in Early Modern Europe (Bologne, Berlin : Il Mulino, Duncker et Humblot, 2012), 303 p.

SAWYER, Jeffrey K., Printed Poison: Pamphlet Propaganda, Faction Politics, and the Public Sphere in Early Seventeenth-Century France (Berkeley: University of California Press, 1990), $178 \mathrm{p}$.

SHEPARD, Alexandra et Phil WITHINGTON, éd., Communities in Early Modern England: Networks, Place, Rhetoric, coll. «Politics, Culture, and Society in Early Modern Britain » (Manchester : Manchester University Press, 2000), $276 \mathrm{p}$.

STARR, Paul, The Creation of the Media : Political Origins of Modern Communications (New York: Basic Books, 2004), « Part 1 - The Opening of the Public Sphere, 1600-1860», 23-151.

STOCK, Brian, The Implications of Literacy: Written Language and Models of Interpretation in the Eleventh and Twelfth Centuries (Princeton: Princeton University Press, 1983), « Textual Communities », 88-240.

SUMMERS, Claude J. et Ted-Larry PEBWORTH, éd., Literary Circles and Cultural Communities in Renaissance England (Columbia : University of Missouri Press, 2000), 243 p.

VANHAELEN, Angela et Joseph P. WARD, éd., Making Space Public in Early Modern Europe. Performance, Geography, Privacy, coll. « Routledge Studies in Renaissance Literature and Culture » (New York, Londres : Routledge, 2013), 305 p.

WARNER, Michael, Publics and Counterpublics (New York, Cambridge : Zone Books, MIT Press, 2002), $334 \mathrm{p}$.

WILSON, Bronwen et Paul Edward YACHNIN, éd., Making Publics in Early Modern Europe : People, Things, Forms of Knowledge, coll. "Routledge Studies in Renaissance Literature and Culture » (New York : Routledge, 2010), $282 \mathrm{p}$.

YACHNIN, Paul Edward et Marlene EBERHART, éd., Forms of Association : Making Publics in Early Modern Europe, coll. " Massachusetts Studies in 
Early Modern Culture » (Amherst : University of Massachusetts Press, 2015), 334 p.

\section{Antagonisme et culture}

Les études ici rassemblées pensent la fécondité des oppositions intergroupes et des concurrences intragroupes sous l'Ancien Régime. Elles empruntent largement aux travaux fondateurs de Georg Simmel sur le conflit (1955) et de Pierre Bourdieu sur le champ littéraire (1991). Important aussi est le texte de Leo Strauss (2009 [1941]) sur les stratégies d'écriture en temps de persécution, où les publications dissidentes s'adressent "non pas à tous les lecteurs, mais seulement au lecteur intelligent et digne de foi " (p. 55). Pour une recension sur l'évolution des travaux autour de Strauss, nous renvoyons le lecteur à la bibliographie en ligne de JeanPierre Cavaillé, citée ci-dessous.

La recherche allemande des dernières décennies sur la «culture du conflit» - «culture of contention » (Streitkultur) - s'est révélée particulièrement prolifique (voir Haugen 2016). Sur le territoire anglais, signalons les travaux récents de Lines, Laureys et Kraye (2015) menés dans le cadre du projet Renaissance Conflict and Rivalries : Cultural Polemics in Europe, c. 1300-c. 1650, dirigé par David Lines (University of Warwick, 2012-2015). Hormis la conflictualité de la République des Lettres, les thèmes de prédilection touchent les rivalités entre les traditions scolastique et humaniste ainsi que la réorganisation des savoirs à la Renaissance. Sur cette dernière question, deux projets de recherche d'envergure ont récemment été entrepris : Creating a Knowledge Society in a Globalizing World (1450-1800), dirigé par Sven Dupré et Wijnand Mijnhardt (Utrecht University), et Worlds and Networks of Higher Learning (1400-1750), sous la direction d'Anja-Silvia Goeing (Northumbria University), Glyn Parry (University of Roehampton) et Mordechai Feingold (California Institute of Technology).

Les publications retenues ne s'attachent ni à une personnalité singulière ni à un genre littéraire précis, à quelques exceptions près, comme l'étude de Béatrice Périgot (2005) sur les transformations de la disputatio à la Renaissance et les travaux d'Amaury Flèges (1994) sur la forme collective du tombeau poétique, envisagé comme lieu d'expression d'un champ littéraire en formation. Le vaste corpus ressortissant à la Querelle des femmes constitue également un témoin important de la "culture du conflit». Loin de se résumer à l'opposition entre les sexes, les rivalités mises en jeu dans cette tradition littéraire forment un tableau 
complexe qu'a notamment étudié Éliane Viennot (2004, 2013). Mentionnons enfin la possibilité d'appréhender l'antagonisme des publics au moyen de la dialectique vainqueurs / vaincus; on consultera alors avec profit le récent collectif dirigé par Jean-Marie Le Gall sur la défaite à la Renaissance (2016).

ALAZARD, Florence, Stéphan GEONGET, Laurent GERBIER et Paul-Alexis MELLET, éd., Dissensus : pratiques et représentations de la diversité des opinions (1500-1650) (Paris : Honoré Champion, 2016), 256 p.

BERRY, Philippa et Margaret TUDEAU-CLAYTON, éd., Textures of Renaissance Knowledge (Manchester, New York : Manchester University Press, 2003), $227 \mathrm{p}$.

BERTHEAU, Gilles et Line COTTEGNIES, éd., Dossier « Figures du conflit dans le théâtre historique européen de la Renaissance ", Études Épistémè. Revue de littérature et de civilisation (XVI $-X V I I I^{e}$ siècles) [en ligne] 14 (2008), URL : https://episteme.revues.org/714.

BONIFAY, Florence et Marjorie BROUSSIN, «Introduction. Courir ensemble vers un même but : la concurrence au cœur du processus de création? ", in Concurrence(s) dans le monde des arts et des lettres, éd. Florence Bonifay, Marjorie Broussin, Stéphane Caruana et Mélanie Guérimand, coll. « Logiques sociales » (Paris : L’Harmattan, 2016), 7-24.

BOULĖGUE, Laurence, Commenter et philosopher à la Renaissance : tradition universitaire, tradition humaniste, coll. «Cahiers de philologie » (Villeneuve d'Ascq : Presses universitaires du Septentrion, 2014), $312 \mathrm{p}$.

BOURDIEU, Pierre, "Le champ littéraire », Actes de la recherche en sciences sociales 89 (1991) : 3-46.

BREMER, Kai et Carlos SPOERHASE, éd., Gelehrte Polemik: Intellektuelle Konfliktverschärfungen um 1700, coll. «Zeitsprünge » (Francfort-sur-leMain : Klostermann, 2011), 440 p.

CAVAILLÉ, Jean-Pierre, «Bibliographie : Mensonge, tromperie, simulation et dissimulation ", Les Dossiers du Grihl [en ligne], 2012, « Philosophie et art d'écrire (autour de Leo Strauss) », URL : http://journals.openedition. org/dossiersgrihl/2103.

CHARBONNEAU, Frédéric, éd., Histoire et conflits, actes du colloque du Cercle interuniversitaire d'étude sur la République des Lettres (CIERL), 16 mai 2006 (Université McGill), coll. «Cahiers du CIERL » (Québec : Les Presses de l'Université Laval, 2007), 102 p. 
CUMMINS, Stephen et Laura KOUNINE, Cultures of Conflict Resolution in Early Modern Europe (Farnham, Burlington : Ashgate, 2016), 292 p.

DUBOIS-NAYT, Armel, Nicole DUFOURNAUD et Anne PAUPERT, éd., Revisiter la "querelle des femmes ». Discours sur l'égalité/inégalité des sexes, Vol. 3 : de 1400 à 1600, coll. «École du genre » (Saint-Étienne : Publications de l'Université de Saint-Étienne, 2013), 282 p.

DUBOIS-NAYT, Armel, Marie-Élisabeth HENNEAU et Rotraud VON KULESSA, éd., Revisiter la "querelle des femmes ". Discours sur l'égalité/inégalité des sexes, Vol. 4 : en Europe, de 1400 aux lendemains de la Révolution, coll. «École du genre » (Saint-Étienne : Publications de l’Université de Saint-Étienne, 2016), 328 p.

FLEGES, Amaury, " "Je ravie le mort”, tombeaux littéraires en France à la Renaissance ", La Licorne 29 (1994) : 71-142.

GAVOILlE, Élisabeth et François GUILLAUMONT, éd., Conflits et polémiques dans l'épistolaire, coll. "Perspectives littéraires » (Tours : Presses universitaires François-Rabelais, 2015), 541 p.

GOUWENS, Kenneth et Christopher S. CELENZA, "Humanist Culture and Its Malcontents : Alcionio, Sepúlveda, and the Consequences of Translating Aristotle ", in Humanism and Creativity in the Renaissance, éd. Christopher S. Celenza et Kenneth Gouwens, coll. « Brill's Studies in Intellectual History » (Leyde, Boston : Brill, 2006), 347-380.

GRAFTON, Anthony, Defenders of the Text: The Traditions of Scholarship in an Age of Science, 1450-1800 (Cambridge : Harvard University Press, 1991), $330 \mathrm{p}$.

GRAFTON, Anthony et Lisa JARDINE, From Humanism to the Humanities : Education and the Liberal Arts in Fifteenth-and Sixteenth-Century Europe (Cambridge : Harvard University Press, 1986), 224 p.

GRAFTON, Anthony et Nancy SIRAISI, Natural Particulars : Nature and the Disciplines in Renaissance Europe, coll. "Dibner Institute Studies in the History of Science and Technology» (Cambridge : MIT Press, 1999), $426 \mathrm{p}$.

HAUGEN, Kristine Louise, "Controversy, Competition, and Insult in the Republic of Letters ", History of Humanities 1.2 (2016) : 399-407.

KELLEY, Donald R., History and the Disciplines : The Reclassification of Knowledge in Early Modern Europe (Rochester : University of Rochester Press, 1997), 344 p. 
LAJARTE, Philippe de, "De la spécificité du champ littéraire au XVI siècle : hypothèses de travail ", in Les Méthodes du discours critique dans les études seiziémistes, actes du Colloque de la S.F.D.S., éd. Gisèle Mathieu-Castellani et Jean-Claude Margolin (Paris : Société d'édition d'enseignement supérieur, 1987), 11-22.

LANDTSHEER, Jeanine et Henk NELLEN, éd., Between Scylla and Charybdis : Learned Letter Writers Navigating the Reefs of Religious and Political Controversy in Early Modern Europe, coll. "Brill's Studies in Intellectual History » (Leyde : Brill, 2011), 539 p.

LAUREYS, Marc et Roswitha SIMONS, éd., The Art of Arguing in the World of Renaissance Humanism (Louvain : Leuven University Press, 2013), 232 p.

LE GALL, Jean-Marie, éd., La Défaite à la Renaissance, coll. "Cahiers d'Humanisme et Renaissance» (Genève : Droz, 2016), 376 p.

LINES, David A., "From Schools to Courts ", in Rethinking Virtue, Reforming Society: New Directions in Renaissance Ethics, c. 1350-c. 1650, éd. David A. Lines et Sabrina Ebbersmeyer, coll. «Cursor mundi » (Turnhout : Brepols, 2013), 57-79.

LINES, David A., "Humanism and the Italian Universities ", in Humanism and Creativity in the Renaissance, éd. Christopher S. Celenza et Kenneth Gouwens, coll. « Brill's Studies in Intellectual History » (Leyde, Boston : Brill, 2006), 327-346.

LINES, David A., Marc LAUREYS et Jill KRAYE, éd., Forms of Conflict and Rivalries in Renaissance Europe (Göttingen : Bonn University Press, V \& R unipress, 2015), $281 \mathrm{p}$.

MARINO, John A. et Thomas KUEHN, éd., A Renaissance of Conflicts. Visions and Revisions of Law and Society in Italy and Spain (Toronto : Centre for Reformation and Renaissance Studies, 2004), $440 \mathrm{p}$.

PANTIN, Isabelle et Gérald PEOUX, éd., Mise en forme des savoirs à la Renaissance. À la croisée des idées, des techniques et des publics (Paris : Armand Colin, 2013), $215 \mathrm{p}$.

PÉRIGOT, Béatrice, Dialectique et littérature: les avatars de la dispute entre Moyen Âge et Renaissance, coll. «Bibliothèque littéraire de la Renaissance » (Paris : Honoré Champion, 2005), $736 \mathrm{p}$.

PETTEGREE, Andrew, The Book in the Renaissance (New Haven : Yale University Press, 2010), « Conflict », 201-270. 
ROBERT, Valérie, « Polémiques entre intellectuels : pratiques et fonctions », in Intellectuels et polémiques dans l'espace germanophone, éd. Valérie Robert, coll. «PIA (Publications de l'Institut d'Allemand) » (Paris : Presses Sorbonne Nouvelle, 2003), 11-59.

RUMMEL, Erika, Biblical Humanism and Scholasticism in the Age of Erasmus, coll. «Brill's Companions to the Christian Tradition » (Leyde, Boston : Brill, 2008), $334 \mathrm{p}$.

SIMMEL, Georg, Conflict and the Web of Group-Affiliations, trad. Kurt H. Wolff et Reinhard Bendix (Glencoe : Free Press, 1955), 195 p.

SIRAISI, Nancy G., éd., Science and Universities of Early Modern Europe : Teaching, Specialization, Professionalization, Early Science and Medicine 6.4 (2001), $384 \mathrm{p}$.

STACEY, Sarah Alyn et Véronique DESNAIN, éd., Culture and Conflict in Seventeenth-Century France and Ireland (Dublin : Four Courts Press, 2004), $288 \mathrm{p}$.

STAYER, James M., «Radicalism and Dissent. A Provisional Assessment », in Radikalität und Dissent im 16. Jahrhundert / Radicalism and Dissent in the Sixteenth Century, éd. Hans-Jürgen Goertz et James M. Stayer, coll. «Zeitschrift für historische Forschung (Berlin : Duncker and Humblot, 2002), 9-27.

STRAUSS, Leo, La Persécution et l'art d'écrire, trad. Olivier Sedeyn, postface d'Arnaldo Momigliano, coll. «Tel » (Paris : Gallimard, 2009), 364 p.

VIALA, Alain, Naissance de l'écrivain. Sociologie de la littérature à l'âge classique, coll. "Sens commun» (Paris : Éditions de Minuit, 1985), 319 p.

VIENNOT, Éliane, "Champions des dames et misogynes : les enjeux d'un combat frontal, à l'aube des temps modernes (France, 1380-1530) ", in L'Engagement des hommes pour l'égalité des sexes (XIVe-XXI siècle), éd. Florence Rochefort et Éliane Viennot, coll. «École du genre » (SaintÉtienne : Publications de l’Université de Saint-Étienne, 2013), 21-36. , "Culture philogyne, culture misogyne : un conflit de classe au cœur de la construction de l'État moderne ", in Culture d'élite, culture de masse et différence des sexes, éd. Geneviève Sellier et Éliane Viennot, coll. «Bibliothèque du féminisme » (Paris : L'Harmattan, 2004), 19-45.

VINESTOCK, Elizabeth et David FOSTER, éd., Writers in Conflict in Sixteenth-Century France : Essays in Honour of Malcolm Quainton, 
coll. «Durham Modern Languages Series» (Durham: Durham University, 2008), 390 p.

WAQUET, Françoise, «La République des Lettres : un univers de conflits », in Pouvoirs, contestations et comportements dans l'Europe moderne. Mélanges en l'honneur d'Yves-Marie Bercé, éd. Bernard Barbiche, Jean-Pierre Poussou et Alain Tallon (Paris : Presses de l'Université Paris-Sorbonne, 2005), 829-840.

, " "Peut-il y avoir des intellectuels sans polémiques ?" La réponse de la République des Lettres ", in Intellectuels et polémiques dans l'espace germanophone, éd. Valérie Robert, coll. « PIA (Publications de l'Institut d'Allemand)» (Paris : Presses Sorbonne Nouvelle, 2003), 377-382.

WIESENFELDT, Gerhard, « Different Modes of Competition ? Early Modern Universities and Their Rivalries ", NTM Zeitschrift für Geschichte der Wissenschaften, Technik und Medizin 24.2 (2016) : 125-139.

\section{Publics confessionnels}

Les trois prochaines sections envisagent les publics en tant que groupes fondés sur des oppositions manifestes : l'allégeance religieuse est ici traitée, suivie de la situation géo-/sociopolitique et linguistique (section 4), puis des distinctions fondées sur le sexe (section 5). Les travaux sur les publics sous la Réformation sont largement tributaires de la notion de confessionnalisation de Heinz Schilling et Wolfgang Reinhard, dont nous incluons plusieurs critiques et états de la question.

BRIETZ MONTA, Susannah, Martyrdom and Literature in Early Modern England (Cambridge, New York : Cambridge University Press, 2005), "Too Many Brides : The Interpretive Community and Ecclesiastical Controversy ", 35-52.

DEVENTER, Jörg, « "Confessionalisation” - A Useful Theoretical Concept for the Study of Religion, Politics, and Society in Early Modern East-Central Europe ? ", Europea Review of History / Revue européenne d'Histoire 11.3 (2004) : 403-425.

FRIEDRICH, Markus, «Les conflits théologiques aux XVI et XVII ${ }^{e}$ siècles : déroulement et dimensions ", in Intellectuels et polémiques dans l'espace germanophone, éd. Valérie Robert, coll. «PIA (Publications de l'Institut d'Allemand)» (Paris : Presses Sorbonne Nouvelle, 2003), 135-144. 
GILMONT, Jean-François, "Les circuits européens du livre réformé au $\mathrm{XVI}^{e}$ siècle ", in Le Livre voyageur : constitution et dissémination des collections livresques dans l'Europe moderne (1450-1830), éd. Dominique Bougé-Grandon (Paris : Klincksieck, 2000), 109-128.

GILMONT, Jean-François et William KEMP, Le Livre évangélique en français avant Calvin / The French Evangelical Book Before Calvin, coll. " Nugæ humanisticæ »(Turnhout, Bruxelles : Brepols, Musée de la Maison d’Érasme, 2004), 391 p.

HEADLEY, John M., Hans J. HILLERBRAND et Anthony H. PAPALAS, éd., Confessionalization in Europe, 1555-1700 (Aldershot, Burlington : Ashgate, 2004), $369 \mathrm{p}$.

HIGMAN, Francis M., Piety and the People: Religious Printing in French, 15111551, coll. «St. Andrews Studies in Reformation History " (Aldershot : Scolar Press, 1996), 534 p.

, Censorship and the Sorbonne: A Bibliographical Study of Books in French Censured by the Faculty of Theology of the University of Paris, 1520-1551, coll. « Travaux d'humanisme et Renaissance » (Genève : Droz, 1979), $199 \mathrm{p}$.

HOUDARD, Sophie, Adelisa MALENA et Xenia von TIPPELSKIRCH, éd., Langages dissidents : performances et contestations religieuses à l'époque moderne, Études Épistémè. Revue de littérature et de civilisation (XVI $I^{e} X V I I I^{e}$ siècles) [en ligne] 31 (2017), URL : https://episteme.revues. org/1506.

JOSTOCK, Ingeborg, La Censure négociée. Le contrôle du livre à Genève, 15601625, coll. "Travaux d'humanisme et Renaissance " (Genève : Droz, 2007), $440 \mathrm{p}$.

KARREMANN, Isabel, Cornel ZWIERLEIN et Inga Mai GROOTE, éd., Forgetting Faith ? Negotiating Confessional Conflict in Early Modern Europe, coll. « Pluralisierung \& Autorität » (Berlin : De Gruyter, 2012), $287 \mathrm{p}$.

LOTZ-HEUMANN, Ute, "The Concept of "Confessionalization" : A Historiographical Paradigm in Dispute », MyC (Memoria y Civilizacion) 4 (2001) : 93-114.

LURIA, Keith, Sacred Boundaries : Religious Co-existence and Conflict in EarlyModern France (Washington : Catholic University of America Press, 2005), $357 \mathrm{p}$. 
MACLEAN, Ian, Scholarship, Commerce, Religion: The Learned Book in the Age of Confessions, 1560-1630 (Cambridge : Harvard University Press, 2012), $380 \mathrm{p}$.

PETTEGREE, Andrew, The French Book and the European Book World (Leyde, Boston : Brill, 2007), 328 p.

, Reformation and the Culture of Persuasion (Cambridge : Cambridge University Press, 2005), $237 \mathrm{p}$.

REID, Jonathan A., King's Sister - Queen of Dissent : Marguerite of Navarre (1492-1549) and her Evangelical Network, coll. «Studies in Medieval and Reformation Traditions» (Leyde, Boston : Brill, 2009), 795 p.

REINHARD, Wolfgang, Papauté, confessions, modernité, coll. "Recherches d'histoire et de sciences sociales " (Paris : Éditions de l'École des hautes études en sciences sociales, 1998), $260 \mathrm{p}$.

SCHILLING, Heinz, «La "Confessionnalisation”, un paradigme comparatif et interdisciplinaire. Historiographie et perspectives de recherche », Études germaniques 57.3 (2002) : 401-420.

, «Confessionalization : Historical and Scholarly Perspectives of a Comparative and Interdisciplinary Paradigm ", in Confessionalization in Europe, 1555-1700. Essays in Honor of Bodo Nischan, éd. John Headley et Hans Hillerbrand (Aldershot: Ashgate, 2004), 21-35.

SCHILLING, Heinzet István György TÓTH, «From Empires to Family Circles: Religious and Cultural Borderlines in the Age of Confessionalisation », in Cultural Exchange in Early Modern Europe, Vol. 1 : Religion and Cultural Exchange in Europe, 1400-1700, éd. Heinz Schilling et István György Tóth (Cambridge : Cambridge University Press, 2007), 25-46.

VAN DUSSEN, Michael et Pavel SOUKUP, «Textual Controversies, Textual Communities ", in Religious Controversy in Europe (1378-1536) : Textual Transmission and Networks of Readership, éd. Michael Van Dussen et Pavel Soukup, coll. " Medieval Church Studies » (Turnhout : Brepols, 2013), 1-16.

\section{Publics géo-/sociopolitiques et groupes linguistiques}

Les communautés étudiées dans la présente section sont circonscrites sur la base de critères intimement liés à la nation (le territoire, l'allégeance politique, la langue). Aussi pourrait-on ajouter à cette liste l'ouvrage de Benedict Anderson, qui figure 
dans la première partie de la bibliographie (Imagined Communities, 2016 [1983]). La conflictualité des publics peut résulter du bras de fer diplomatique entre grands états (voir notamment Hampton 2009) ou encore, au sein d'une même nation, du recoupement imparfait entre un espace politique et un corps idéologique, par exemple entre "cités humanistes » et « cités politiques " (CrouzetPavan, Crouzet et Desan 2014). Sur la question linguistique, nous avons privilégié les travaux qui portent sur une forme quelconque de tension, par exemple la "marginalisation et résistance des langues de France » (Courouau 2012) et les rivalités entre traducteurs (Duché et Uetani 2015).

BISTUÉ, Belén, Collaborative Translation and Multi-Version Texts in Early Modern Europe, coll. "Transculturalisms, 1400-1700 " (Burlington : Ashgate, 2013), $183 \mathrm{p}$. , «The Task(s) of the Translator(s): Multiplicity as Problem in Renaissance European Thought », Comparative Literature Studies 48.2 (2011) : 139164.

BREITENSTEIN, Renée-Claude et Tristan ViglianO, éd., Le Choix de la langue dans la construction des publics en France à la Renaissance, actes du colloque de l'Université Brock, Le Français préclassique 14 (2012), 244 p. BURKE, Peter, «The Renaissance Translator as Go-Between », in Renaissance Go-Betweens : Cultural Exchange in Early Modern Europe, éd. Andreas Höfele et Werner von Koppenfels (Berlin, New York : Walter de Gruyter, 2005), 17-31.

, Languages and Communities in Early Modern Europe (Cambridge : Cambridge University Press, 2004), 210 p.

CANTAGREL, Laurent, Discours lettré et transformations sociopolitiques au début du XVI siècle, coll. "Études et essais sur la Renaissance » (Paris : Classiques Garnier, 2012), 336 p.

CORDINGLEY, Anthony et Céline FRIGAU MANNING, éd., Collaborative Translation. From the Renaissance to the Digital Age, coll. «Bloomsbury Advances in Translation » (Londres, New York : Bloomsbury Academic, 2017), $260 \mathrm{p}$.

COUROUAU, Jean-François, Et non autrement : marginalisation et résistance des langues de France (XVI ${ }^{e}-X V I I^{e}$ siècle), coll. « Cahiers d'Humanisme et Renaissance » (Genève : Droz, 2012), 291 p. 
, Moun lengatge bèl : les choix linguistiques minoritaires en France : 14901660, coll. «Cahiers d'Humanisme et Renaissance » (Genève : Droz, 2008), 472 p.

CROUZET-PAVAN, Élisabeth, Denis CROUZET et Philippe DESAN, éd., Cités humanistes, cités politiques (1400-1600) (Paris : Presses de l’Université Paris-Sorbonne, 2014), 316 p.

DAVIES, Surekha, Renaissance Ethnography and the Invention of the Human: New Worlds, Maps, and Monsters, coll. "Cambridge Social and Cultural Press » (New York : Cambridge University Press, 2016), 348 p.

DUBARD, Frédérique et Davide LUGLIO, éd., Les "Rivales latines ». Lieux, modalités et figures de la confrontation franco-italienne, Revue des études italiennes 59 (2013), 304 p.

DUCHÉ, Véronique et Toshinori UETANI, avec la collab. de Susan BADDELEY et Jean-Marc DECHAUD, «Collaborations, rivalités et cabales ", in Histoire des traductions en langue française, XV $V^{e}$ et XVI siècles (1470-1610), éd. Véronique Duché (Lagrasse : Verdier, 2015), 388-405.

GAD OFFRE, Gilbert, La Révolution culturelle dans la France des Humanistes : Guillaume Budé et François ${ }^{2}$, coll. "Titre courant » (Genève : Droz, 1997), $349 \mathrm{p}$.

GIACOMOTTO-CHARRA, Violaine et Christine SILVI, éd., Lire, choisir, écrire : la vulgarisation des savoirs du Moyen Âge à la Renaissance (Paris : École des chartes, 2014), 274 p.

GRUTMAN, Rainier, « Frontières et négociations linguistiques à la Renaissance », Le Français préclassique 14 (2012) : 213-229.

HAMPTON, Timothy, Fictions of Embassy : Literature and Diplomacy in Early Modern Europe (Ithaca : Cornell University Press, 2009), 235 p. , «The Diplomatic Moment : Representing Negotiation in Early Modern Europe », Modern Language Quarterly 57.1 (2006) : 81-102. , Literature and Nation in the Sixteenth Century. Inventing Renaissance France (Ithaca : Cornell University Press, 2001), 289 p.

HELMERS, Helmer, «Public Diplomacy in Early Modern Europe », Media History 22.3-4 (2016) : 401-420.

HIRSCHI, Caspar, "Höflinge der Bürgerschaft - Bürger des Hofes. Zur Beziehung von Humanismus und städtischer Gesellschaft ", in Humanismus und Renaissance in Augsburg. Kulturgeschichte einer Stadt 
zwischen Spätmittelalter und Dreißigjährigem Krieg, éd. Gernot Michael Müller (Berlin, New York: De Gruyter, 2010), 31-60.

KELLER, Vera, « Painted Friends: Political Interest and the Transformation of International Learned Sociability ", in Friendship in the Middle Ages and Early Modern Times, éd. Marilyn Sandidge et Albrecht Classen (Berlin : De Gruyter, 2011), 661-692.

MIGNOLO, Walter, The Darker Side of the Renaissance : Literacy, Territoriality, and Colonization (Ann Arbor : University of Michigan Press, 2003 [1995]), $463 \mathrm{p}$.

PERRY, Curtis, Literature and Favoritism in Early Modern England (Cambridge, New York : Cambridge University Press, 2006), 328 p.

RAMAN, Shankar, Renaissance Literature and Postcolonial Studies, coll. « Postcolonial Literary Studies » (Édimbourg : Edinburgh University Press, 2011), $186 \mathrm{p}$.

WARD, Joseph P., Metropolitan Communities : Trade Guilds, Identity, and Change in Early Modern London (Stanford : Stanford University Press, 1997), $203 \mathrm{p}$.

\section{Publics féminins}

L'objectif de cette section est de présenter les communautés renaissantes de lectrices et leurs inscriptions textuelles à la fois dans leur diversité et leur spécificité. On trouvera donc autant des considérations générales sur la littératie des femmes sous l'Ancien Régime que des réflexions sur les publics féminins d'un genre spécifique (le recueil de nouvelles, les amours, etc.), d'un auteur (Montaigne, Louise Labé, etc.) ou d'une cuvre (l'Heptaméron, les Rymes, etc.). On se reportera plutôt à la sixième et dernière section pour les recherches sur la direction éditoriale des femmes libraires au XVI siècle, groupe social ayant suscité l'intérêt des spécialistes de l'histoire matérielle du livre (Arbour 1997, Jimenes 2017). Les travaux listés portent sur une production littéraire d'expression française, hormis quelques ouvrages de référence dressant un état de la recherche sur l'histoire des lectrices en Angleterre (essentiellement Brayman Hackel 2005 et 2010), en Italie (Tippelskirch 2007), en Espagne et en Nouvelle Espagne (De Courcelles et Val Juliàn 1999). Enfin, notons que la présente section entretient un lien évident avec les études portant sur la Querelle des femmes, abordées plus haut dans le cadre plus général de la «culture du conflit». 
ALDUY, Cécile, «Lecteurs réels et lectrices alléguées : lire les recueils d'Amours au XVI ${ }^{\mathrm{e}}$ siècle ", La lecture littéraire. Revue de recherche sur la lecture des textes littéraires 7 (2003) : 119-121.

BALSAMO, Jean, « Montaigne et ses lectrices », Revue d'études culturelles 3 (2007) : 71-83.

BAUSCHATZ, Cathleen M., " "Voylà, mes dames...”. Inscribed Women Listeners and Readers in the Heptameron ", in Critical Tales. New Studies of the Heptameron and Early Modern Culture, éd. John D. Lyons et Mary B. McKinley (Philadelphia : University of Pennsylvania Press, 1993), 103122.

BERRIOT-SALVADORE, Évelyne, "Les héritières de Louise Labé », in Louise Labé: les voix du lyrisme, éd. Guy Demerson (Paris, Saint-Étienne : Éditions du CNRS, Publications de l'Université de Saint-Étienne, 1990), 93-106.

BERRIOT-SALVADORE, Évelyne, Les Femmes dans la société française de la Renaissance, coll. " Histoire des idées et critique littéraire » (Genève : Droz, 1990), $592 \mathrm{p}$.

BLAISDELL, Charmarie, «Religion, Gender, and Class : Nuns and Authority in Early Modern France ", in Changing Identities in Early Modern France, éd. Michael Wolfe (Durham : Duke University Press, 1997), 147-168.

BRAYMAN HACKEL, Heidi, «Reading Women », in The History of British Women's Writing, 1500-1610, éd. Caroline Bicks et Jennifer Summit, coll. « History of British Women's Writing » (New York : Palgrave Macmillan, 2010), 17-33.

, Reading Material in Early Modern England: Print, Gender, and Literacy (Cambridge : Cambridge University Press, 2005), 322 p.

BRAYMAN HACKEL, Heidi et Catherine E. KELLY, éd., Reading Women : Literacy, Authorship, and Culture in the Atlantic World, 1500-1800, coll. « Material Texts» (Philadelphie : University of Pennsylvania Press, 2008), $263 \mathrm{p}$.

BREITENSTEIN, Renée-Claude, «Tensions fécondes dans la construction de publics féminins à l'aube de la Renaissance : les exemples de La Nef des dames vertueuses de Symphorien Champier et de La Louenge de mariage et recueil des hystoires des bonnes, vertueuses et illustres femmes de Pierre de Lesnauderie ", in Les Femmes, la culture et les arts en Europe, éd. 
Cynthia J. Brown et Anne-Marie Legaré, coll. « Texte, codex et contexte » (Turnhout : Brepols, 2016), 241-257.

BROMILOW, Pollie, «An Emerging Female Readership of Print in SixteenthCentury France ? Pierre de Changy's Translation of the De institutione femince Christiance by Juan Luis Vives », French Studies 67.2 (2013) : 155169.

BROOMHALL, Susan et Stephanie TARBIN, éd., Women, Identities and Communities in Early Modern Europe, coll. "Women and Gender in the Early Modern World» (Aldershot, Burlington : Ashgate, 2008), 242 p.

BROUARD-ARENDS, Isabelle, éd., Lectrices d'Ancien Régime, coll. «Interférences» (Rennes : Presses Universitaires de Rennes, 2003), 719 p.

BUNG, Stephanie, «Topiques de la voix. Conversation vs éloquence dans les salons de l'Ancien Régime ", in Femmes, rhétorique et éloquence sous l'Ancien Régime, éd. Claude La Charité et Roxanne Roy, coll. «École du genre » (Saint-Étienne : Publications de l'Université de Saint-Étienne, 2012), 333-343.

BURKE, Victoria E., «Reading Friends : Women's Participation in "masculine" literary culture ", in Early Modern Women's Manuscript Writing, éd. Victoria E. Burke et Jonathan Gibson (Farnham, Burlington : Ashgate, 2004), 75-90.

BUTTERWORTH, Emily, " Gossip and the Public Sphere in Les Caquets de l'Accouchée (1622) », Forum for Modern Language Studies 50.2 (2014) : 142-153.

CAMPBELL, Julie D., Literary Circles and Gender in Early Modern Europe, coll. "Women and Gender in the Early Modern World » (Farnham, Burlington : Ashgate, 2006), $221 \mathrm{p}$.

CAMPBELL, Julie D. et Anne R. LARSEN, éd., Early Modern Women and Transnational Communities of Letters, coll. "Women and Gender in the Early Modern World» (Farnham : Ashgate, 2009), 330 p.

CARR, Thomas M., éd., "A Checklist of Published Writings in French by Early Modern Nuns », EMF : Studies in Early Modern France 11 (2007): 231-257.

CHANG, Leah, Into Print: The Production of Female Authorship in Early Modern France (Newark : University of Delaware Press, 2009), 284 p.

CLARK-EVANS, Christine, "On the Communion of Women : Reading and Writing in the Poetry of Pernette du Guillet and Louise Labé », Proceedings 
of the PMR Conference: Annual Publication of the International Patristic, Mediaeval and Renaissance Conference 12-13 (1987-1988) : 67-80.

CLÉMENT, Michèle et Janine INCARDONA, éd., L'Émergence littéraire des femmes à Lyon à la Renaissance, 1520-1560, coll. «École du genre » (SaintÉtienne: Publications de l'Université de Saint-Étienne, 2008), 284 p.

COURCELlES, Dominique de et Carmen VAL JULIÁN, "Bibliothèques de femmes : identités sociales et génériques » et «Un espace féminin des livres et de la lecture ? ", in Des Femmes et des livres. France et Espagne, $X I V^{e}-X V I I^{e}$ siècle, actes de la journée d'études organisée par l'École nationale des Chartes et l'École Normale Supérieure de Fontenay-SaintCloud (Paris, 30 avril 1998), éd. Dominique de Courcelles et Carmen Val Julián, coll. «Études et rencontres de l'École des Chartes » (Paris : École des Chartes, 1999), 7-77 et 79-115.

CRAWFORD, Julie, «Literary Circles and Coteries ", in The History of British Women's Writing, 1500-1610, éd. Caroline Bicks et Jennifer Summit, coll. « History of British Women's Writing » (New York : Palgrave Macmillan, 2010), 34-59.

DEZUR, Kathryn, "Vaine books" and Early Modern Women Readers ", in Reading and Literacy in the Middle Ages and Renaissance, éd. Ian Frederick Moulton, coll. " Arizona Studies in the Middle Ages and the Renaissance » (Turnhout: Brepols, 2004), 105-125.

FERGUSON, Margaret W., Dido's Daughters : Literacy, Gender and Empire in Early Modern England and France (Chicago : University of Chicago Press, 2003), $506 \mathrm{p}$.

FERGUSON, Margeret W. et Mihoko SUZUKI, «Women's Literacies and Social Hierarchy in Early Modern England », Literature Compass 12.11 (2015) : 575-590.

GOLDSMith, Elizabeth C. et Dena GOOdMAN, Going Public: Women and Publishing in Early Modern France (Ithaca : Cornell University Press, 1995), $249 \mathrm{p}$.

GRANDE, Nathalie, «Claude Barbin, un libraire pour dames ? ", Revue de la Bibliothèque nationale de France 39 (2011) : 23-27.

JONES, Ann Rosalind, « Contentious Readings. Urban Humanism and Gender Difference in La Puce de Madame Des-Roches (1582) », Renaissance Quarterly 48.1 (1995) : 109-128. 
JONES, Ann Rosalind, «City Women and their Audiences : Louise Labé and Veronica Franco ", in Rewriting the Renaissance: The Discourses of Sexual Difference in Early Modern Europe, éd. Margaret W. Ferguson, Maureen Quilligan et Nancy J. Vickers, coll. "Women in Culture and Society " (Chicago : University of Chicago Press, 1986), 299-316.

LA CHARITÉ, Claude, " "Ce male vers enfant de ta verve femelle". Les destinataires féminins de la lyrique amoureuse de Marie de Romieu ", Nouvelle Revue du XVI Siècle 18.2 (2000) : 79-92.

LAMB, Mary Ellen, "Constructions of Women Readers », in Teaching Tudor and Stuart Women Writers, éd. Susanne Woods et Margaret P. Hannay, coll. « Options for Teaching» (New York: Modern Language Association, 2000), 23-34.

LARSEN, Anne R., "A Women's Republic of Letters. Anna Maria van Schurman, Marie de Gournay and Female Self-Representation in Relation to the Public Sphere », Early Modern Women 3 (2008) : 105-126.

LAZARD, Madeleine, Les Avenues de Fémynie: les femmes et la Renaissance (Paris : Fayard, 2001), «Les salons des dames », 291-308.

LEG ARÉ, Anne-Marie, éd., Livres et lectures de femmes en Europe entre Moyen âge et Renaissance (Turnhout : Brepols, 2007), 378 p.

LlEWELlYN, Kathleen M., Representing Judith in Early Modern French Literature (Farnham / Burlington : Ashgate, coll. «Women and Gender in the Early Modern World », 2014), "Chantant avec Judith : Establishing Community in Gabrielle de Coignard's Imitation de la victoire de Judich», 75-94.

, "Afin que vous connaissiez, Mesdames": The Heptameron and Conduct Literature for Women », in Approaches to Teaching Marguerite de Navarre's Heptameron, éd. Colette H. Winn, coll. « Approaches to Teaching World Literature » (New York: The Modern Language Association of America, 2007), 52-56.

LOSSE, Deborah N., "Women Addressing Women », in Renaissance Women Writers : French Texts, American Contexts, éd. Anne R. Larsen et Colette H. Winn (Detroit : Wayne State University Press, 1994), 23-37.

READ, Kirk D., "Women of the French Renaissance in Search of Literary Community : A Prolegomenon to Early Modern Women's Participation in Letters », Romance Languages Annual 5 (1993) : 95-102. 
, French Renaissance Women Writers in Search of Community : Literary Constructions of Female Companionship in City, Family and Convent. Louise Labé, Lionnoize, Madeleine and Catherine des Roches, mère et fille, Anne de Marquets, Sour de Poissy (thèse de doctorat, Princeton University, 1991), $431 \mathrm{p}$.

SHAHAR, Annette, L'Écriture féminine au XVI siècle, en France (Lewiston : Edwin Mellen Press, 2008), «L'essor des salons littéraires », 53-73.

SNOOK, Edith, Women, Reading, and the Cultural Politics of Early Modern England, coll. "Women and Gender in the Early Modern World » (Aldershot, Burlington : Ashgate, 2005), 188 p.

STEDMAN, Gesa et Margarete ZIMMERMANN, éd., Höfe Salons - Akademien : Kulturtransfer und Gender im Europa der Frühen Neuzeit (Hildesheim : Georg Olms Verlag AG, 2007), 372 p.

TARTE, Kendall B., "Early Modern Literary Communities. Madeleine des Roches' City of Women », The Sixteenth Century Journal 35 (2004) : 751769.

TIMMERMANS, Linda, L’Accès des femmes à la culture (1598-1715). Un débat d'idées de Saint François de Sales à la Marquise de Lambert, coll. « Champion classiques » (Paris : Honoré Champion, 2005 [1993]), $967 \mathrm{p}$.

TIPPELSKIRCH, Xenia von, « Histoires de lectrices en Italie au début de l'époque moderne : lecture et genre », Revue de synthèse 6.1-2 (2007) : 181-208.

VIENNOT, Éliane, «Ce que l'imprimerie changea pour les femmes », Revue de la Bibliothèque nationale de France 39 (2011) : 14-21.

, "Les intellectuelles de la Renaissance. Enjeux et conflits d'une émergence ", in Intellectuelles : du genre en histoire des intellectuels, éd. Nicole Racine et Michel Trebitsch, coll. " Histoire du temps présent » (Bruxelles : Complexe, 2004), 43-56.

WHEALE, Nigel, Writing and Society. Literacy, Print and Politics in Britain : 1590-1660 (Londres, New York : Routledge, 1999), " "Dressed up with the Flowers of a Library" : Women Reading and Writing ", 105-137.

WIESNER-HANKS, Merry E., Women and Gender in Early Modern Europe, coll. « New Approaches to European History" (Cambridge : Cambridge University Press, 2008), « Literacy and Learning », 141-173. 
WIESNER-HANKS, Merry E., éd., Mapping Gendered Routes and Spaces in the Early Modern World (Farnham, Burlington : Ashgate, 2015), « Part III : Communities and Networks », 203-270.

WILSON-CHEVALIER, Kathleen et Éliane VIENNOT, éd., avec la collab. de Michel Melot et Céleste Schenck, Royaume de fémynie. Pouvoirs, contraintes, espaces de liberté des femmes, de la Renaissance à la Fronde (Paris : Honoré Champion, 1999), $304 \mathrm{p}$.

WOSHINSKY, Barbara R., Imagining Women's Conventual Spaces in France, 1600-1800 : The Cloister Disclosed, coll. "Women and Gender in the Early Modern World» (Farnham, Burlington : Ashgate, 2010), 344 p.

\section{Monde du livre et lectorats}

Les travaux suivants envisagent le public au sens strict de lectorat. Outre quelques états présents de la recherche sur l'histoire du livre et de la lecture (Bessire 2005, Holtz 2014, Tenger et Trolander 2010, Varry 2014), on y trouve, d'une part, des études sur les stratégies textuelles de destination (ainsi, Jourde et al. 2003, La Charité 2011, Simonin 1999) et, d'autre part, des études sur le marché du livre, qu'il s'agisse de concurrence (Brown 2010, Maclean 2009), de privilège royal (Armstrong 1990, Clément et Keller-Rahbé 2017, Keller-Rahbé 2017), de contrefaçon (Gilmont 2006, Mounier et Nativel 2014), de censure (Farge 1996, McLeod 2011), de la peur de l'« invendu " (Jourde 2007) ou de la figure de l'imprimeur-libraire (Balsamo 2006, Bjaï et Rouget 2015, Bonifay 2013, Gilmont 2005, Keller-Rahbé 2010).

ADELIN FIORATO, Charles et Jean-Claude MARGOLIN, éd., L'Écrivain face à son public en France et en Italie à la Renaissance, actes du colloque international de Tours (4-6 décembre 1986) (Paris : Vrin, 1989), 390 p.

ALCORN BARON, Sabrina, Eric N. LIIND QUIST et Eleanor F. SHEVLIN, éd., Agent of Change : Print Culture Studies After Elizabeth L. Eisenstein, coll. «Studies in Print Culture and the History of the Book» (Amherst: University of Massachusetts Press, 2007), 442 p.

AQUILON, Pierre et Henri-Jean MARTIN, éd., avec la collab. de François DUPUIGRENET DESROUSSILLES, Le Livre dans l'Europe de la Renaissance, actes du XXVIII colloque international d'études humanistes de Tours (Paris : Promodis, 1988), 587 p. 
ARBOUR, Roméo, Les Femmes et les métiers du livre (1600-1650) (Chicago, Paris : Garamond Press, Didier érudition, 1997), $314 \mathrm{p}$.

ARMSTRONG, Elizabeth, Before Copyright. The French Book-privilege System, 1498-1526 (Cambridge : Cambridge University Press, 1990), 317 p.

AZROUMANOV, Anna, Anne RÉACH-NGÔ et Trung TRAN, éd., Le Discours du livre: mise en scène du texte et fabrique de l'ouvre sous l'Ancien Régime, coll. "Études et essais sur la Renaissance " (Paris : Classiques Garnier, 2011), 329 p.

BALSAMO, Jean, "Une institution littéraire de la Renaissance : la "boutique de libraire" ", in L'Écrivain et ses institutions, éd. Roger Marchal, coll. « Travaux de Littérature » (Genève : Droz, 2006), 75-96.

BESSIRE, François, «Penser livre», in L'Histoire littéraire à l'aube du XXIe siècle. Controverses et consensus, éd. Luc Fraisse (Paris : Presses universitaires de France, 2005), 231-240.

BJAÏ, Denis et François ROUGET, éd., Les Poètes français de la Renaissance et leurs «libraires» (Genève : Droz, 2015), 549 p.

BONIFAY, Florence, "Figures d'imprimeurs libraires dans quelques productions poétiques de la Brigade ", Réforme Humanisme Renaissace 76 (2013) : 49-71.

BOZZOLA, Carla et Ezio ORNATO, « Les lectures des Français aux XIV et $\mathrm{XV}^{\mathrm{e}}$ siècles. Une approche quantitative », in Ensi firent li ancessor. Mélanges de philologie médiévale offerts à Marx-René Jung, éd. Luciano Rossi, avec la collab. de Christine Jacob-Hugon et Ursula Bähler (Alexandrie : Éd. dell'Orso, 1996), 713-762.

BREITENSTEIN, Renée-Claude, éd., Publics et publications dans les éloges collectifs de femmes à la fin du Moyen Âge et sous l'Ancien Régime, Études françaises 47.3 (2011), $182 \mathrm{p}$.

BROTTIER, Béatrice, "Le travail du libraire dans les avis au lecteur des recueils collectifs de poésies du début du XVII e siècle ", in La Préface : formes et enjeux d'un discours d'escorte, éd. Marie-Pier Luneau et Denis Saint-Amand, coll. "Rencontres » (Paris : Classiques Garnier, 2016), 209-224.

BROWN, Cynthia J., éd., The Cultural and Political Legacy of Anne de Bretagne: Negotiating Convention in Books and Documents, coll. "Gallica " (Cambridge : D.S. Brewer, 2010), « Part I : The Politics of Bookmaking 
in Fifteenth- and Sixteenth-Century Brittany : Cooperation and Competition between France and the Queen's Duchy », 11-46.

CAVALLO, Guglielmo et Roger CHARTIER, éd., Histoire de la lecture dans le monde occidental, coll. « Points histoire» (Paris : Seuil, 2001), 587 p.

CHARON, Annie, Claire LESAGE et Ève NETCHINE, éd., Le Livre entre le commerce et l'histoire des idées : les catalogues de libraires, $X V^{e}-X I X^{e}$ siècles (Paris : École des Chartes, 2011), 280 p.

CHARTIER, Roger, Culture écrite et société : l'ordre des livres (XIVe-XVIII siècle), coll. «Bibliothèque Albin Michel. Histoire » (Paris : Albin Michel, 1996), « Communautés de lecteurs », 133-154.

, Lectures et lecteurs dans la France d'Ancien Régime, coll. "L'Univers historique» (Paris : Seuil, 1987), $375 \mathrm{p}$.

CHATELAIN, Jean-Marc, La Bibliothèque de l'honnête homme: livres et collections en France à l'âge classique (Paris : Bibliothèque nationale de France, 2003), $211 \mathrm{p}$.

CHATELAIN, Jean-Marc et Bernard TEYSSANDIER, éd., L'Idée des bibliothèques à l'âge classique, Littératures classiques 66 (2008), 222 p.

CLÉMENT, Michèle et Edwige KELLER-RAHBÉ, Privilèges d'auteurs et d'autrices en France (XVI $-X V I I^{e}$ siècles). Anthologie critique (Paris : Classiques Garnier, 2017), 568 p.

COPPENS, Christian, éd., Printers and Readers in the Sixteenth Century: Including the Proceedings from the Colloquium Organised by the Centre for European Culture, 9 June 2000, coll. «Bibliologia » (Bruxelles, Turnhout : AVWK, Brepols, 2005), 517 p.

DEJEAN, Joan E., «The Invention of a Public for Literature », EMF : Studies in Early Modern France 3 (1997) : 149-168.

DELOINCE-LOUETTE, Christiane, " Hélisenne de Crenne, Cardan, Montaigne, Aubigné : destinataires explicites et implicites de l'écriture de soi au $\mathrm{XVI}^{\mathrm{e}}$ siècle ", in Les Destinataires du moi : altérités de l'autobiographie, éd. Shojiro Kuwasa, Makoto Masuda et Jean-Christophe Sampieri (Dijon : Éditions universitaires de Dijon, 2012), 13-27.

FARGE, James K., "Early Censorship of Printed Books in Paris : New Perspectives and Insights ", in Le Contrôle des idées à la Renaissance, actes du colloque de la FISIER (Montréal, septembre 1995), éd. J. M. De Bujanda, coll. «Études de philologie et d'histoire (Genève : Droz, 1996), 75-92. 
FURNO, Martine, Qui écrit ? Figures de l'auteur et des co-élaborateurs du texte, $X V^{e}-X V I I I^{e}$ siècles (Lyon : ENS Éditions, Institut d'Histoire du Livre, 2009), 262 p.

FURNO, Martine et Raphaële MOUREN, éd., Auteur, traducteur, collaborateur, imprimeur, qui écrit ?, coll. «Études et essais sur la Renaissance » (Paris : Classiques Garnier, 2012), 329 p.

GILMONT, Jean-François, "Peut-on parler de "contrefaçons" au XVI et au début du XVII ${ }^{\mathrm{e}}$ siècle ? La situation de Genève et d'ailleurs », Bulletin $d u$ bibliophile 1 (2006) : 19-40. , «Les réalités économiques du métier. Marchands-libraires et imprimeurs au $\mathrm{XVI}^{\mathrm{e}}$ siècle ", L'Erasmo. Trimestrale della civiltà europea 25 (2005) : 4-13.

GIROT, Jean-Eudes, éd., Le Poète et son œuvre: de la composition à la publication, actes du colloque de Valenciennes (20-21 mai 1999), coll. «Études de Philologie et d'Histoire »(Genève : Droz, 2004), 384 p.

GORRIS CAMOS, Rosanna et Alexandre VANAUTGAERDEN, éd., Les Labyrinthes de l'esprit. Collections et bibliothèques à la Renaissance. Renaissance Libraries and Collections (Genève : Droz, 2015), 674 p.

GRAFTON, Anthony, Commerce with the Classics: Ancient Books and Renaissance Readers (Ann Arbor : University of Michigan Press, 1997), $237 \mathrm{p}$.

HARRIGAN, Michael, "Addressing a Public in the Seventeenth Century ", Irish Journal of French Studies 10 (2010) : 19-37.

HOLTZ, Grégoire, "Introduction ", in Nouveaux aspects de la culture de l'imprimé : questions et perspectives (XVe-XVII siècles), éd. Grégoire Holtz, coll. "Cahiers d'Humanisme et Renaissance » (Genève : Droz, 2014), 7-34.

JIMENES, Rémi, Charlotte Guillard : une femme imprimeur à la Renaissance (Rennes, Tours : Presses universitaires de Rennes, Presses universitaires François-Rabelais, 2017), 256 p.

JONES DAVIES, M. T., éd., L'Auteur et son public au temps de la Renaissance (Paris : Klincksieck, 1998), 230 p.

JOURDE, Michel, «L'invendu. Mémoire et oubli dans les représentations de l'avenir des livres au premier siècle de l'imprimerie », Études de lettres 276 (2007) : 291-322. 
JOURDE, Michel, Jean-Yves TILLIETTE, Michèle GALLY et al., « "Va, mon livre" : quelques jalons pour une histoire de la destination ", Nouvelle Revue du XVI e siècle 21.1 (2003) : 121-151.

JUlHE, Jean-Claude, éd., Pratiques latines de la dédicace. Permanence et mutations, de l'Antiquité à la Renaissance, coll. "Colloques, congrès et conférences sur la Renaissance européenne » (Paris : Classiques Garnier, 2014), « Deuxième partie. Moyen Âge et Renaissance : de nouveaux enjeux pour la dédicace? ", 399-584.

KAMERBEEK Jr., Jan, "Le concept du "lecteur idéal” », Neophilologus 61.1 (1977) : 2-7.

KELLER-RAHBÉ, Edwige, éd., Les Arrière-boutiques de la littérature. Auteurs et imprimeurs-libraires aux XVI ${ }^{e}-X V I I^{e}$ siècles, coll. "Cribles XVI ${ }^{e}-X V I I{ }^{e}$ : essais de littérature » (Toulouse : Presses universitaires du Mirail, 2010), $262 \mathrm{p}$.

, éd., Privilèges de librairie en France et en Europe, $X V I^{e}-X V I I^{e}$ siècles (Paris : Classiques Garnier, 2017), 539 p.

KESSON, Andy et Emma SMITH, «Introduction : Towards a Definition of Print Popularity ", in The Elizabethan Top Ten: Defining Print Popularity in Early Modern England, éd. Andy Kesson et Emma Smith, coll. « Material Readings in Early Modern Culture " (Farnham, Burlington : Ashgate, 2013), 1-18.

LA CHARITÉ, Claude, « La construction du public lecteur dans le Recueil des dames de Brantôme et les dédicataires, Marguerite de Valois et François d'Alençon ", Études françaises 47.3 (2011) : 109-126.

LE CADET, Nicolas, " "Beuveurs tresillustres, et vous verolez tresprecieux" : Rabelais et les anagnostes ", Revue d'histoire littéraire de la France 115.2 (2015) : 261-282.

LOTTE ANDERSEN, Jennifer et Elizabeth SAUER, éd., Books and Readers in Early Modern England: Material Studies, coll. "Material Texts" (Philadephia : University of Pennsylvania Press, 2002), 305 p.

LOUGH, John, L'Écrivain et son public. Commerce du livre et commerce des idées en France du Moyen Âge à nos jours, trad. Alexis Tadié, coll. "Le Temps et la mémoire » (Paris : Le Chemin vert, 1987), $383 \mathrm{p}$.

LOWRY, Martin, Le Monde d'Alde Manuce : imprimeurs, hommes d'affaires et intellectuels dans la Venise de la Renaissance (Paris : Promodis, 1989), $355 \mathrm{p}$. 
LYONS, Martyn, A History of Reading and Writing in the Western World (Houndmills : Palgrave MacMillan, 2010), «Renaissance Books and Humanist Readers ", 58-72.

MACLEAN, Ian, Learning and the Market Place: Essays in the History of the Early Modern Book (Leyde, Boston : Brill, 2009), 457 p. , "The Readership of Philosophical Fictions. The Bibliographical Evidence », in Philosophical Fictions and the French Renaissance, éd. Neil Kenny, coll. «Warburg Institute Surveys and Texts » (Londres : Warburg Institute, 1991), 7-15.

MCGRADY, Deborah, Controlling Readers : Guillaume de Machaut and His Late Medieval Audience (Toronto : University of Toronto Press, 2006), "Introduction : Reading and the Laity » et "Inscribed Readers : The Invention of the Lay Reader in Text and Image », 3-16 et 17-76.

MCLEOD, Jane, Licensing Loyalty. Printers, Patrons, and the State in Early Modern France, coll. «The Pennsylvania State Series in the History of the Book» (University Park : The Pennsylvania State University Press, 2011), $302 \mathrm{p}$.

MELLOT, Jean-Dominique et Elisabeth QUEVAL, Répertoire d'imprimeurs libraires, XVI ${ }^{e}-X V I I I^{e}$ siècles (Paris : Bibliothèque nationale de France, 1997), $719 \mathrm{p}$.

MOUNIER, Pascale, "Les antécédents lyonnais de la "Bibliothèque Bleue" au XVI e siècle : la constitution d'un romanesque pour le grand public », Littératures 72 (2015) : 191-216.

MOUNIER, Pascale et Colette NATIVEL, éd., Copier et contrefaire à la Renaissance : faux et usage de faux, actes du colloque organisé par R.H.R. et la S.F.D.E.S., coll. « Colloques, congrès et conférences sur le $\mathrm{XVI}^{\mathrm{e}}$ siècle» (Paris : Honoré Champion, 2014), 491 p.

MOUNIER, Pascale et Marie-Claire THOMINE, "Lecteurs de romans, lecteurs de récits brefs : quelques représentations chez les auteurs $\mathrm{du} \mathrm{XVI}^{e}$ siècle ", in Paroles dégelées : propos de l'Atelier XVI siècle, éd. Isabelle Garnier et al., coll. «Études et essais sur la Renaissance » (Paris : Classiques Garnier, 2016), 515-545.

MURA KAMI, Ineke, Moral Play and Counterpublic. Transformations in Moral Drama (1465-1599) (New York, Londres : Routledge, 2011), 247 p. 
MYERS, Robin, Spreading the Word, the Distribution Networks of Print. 1500-1850 (Winchester : St. Paul's Bibliographies, Oak Knoll Press, 1998 [1990]), $241 \mathrm{p}$.

MYERS, Robin, Michael HARRIS et Giles MANDELBROTE, éd., Books for Sale. The Advertising and Promotion of Print Since the Fifteenth Century, coll. «Publishing Pathways » (New Castle, Londres : Oak Knoll Press, The British Library, 2009), 191 p.

MYERS, Robin, Michael HARRIS et Giles MANDELBROTE, éd., Fairs, Markets and the Itinerant Book Trade, coll. « Publishing Pathways " (New Castle, Londres : Oak Knoll Press, The British Library, 2007), 240 p.

, "Le commerce du livre étranger à Paris au XVI siècle ", in Le Livre voyageur : constitution et dissémination des collections livresques dans l'Europe moderne (1450-1830), éd. Dominique Bougé-Grandon (Paris : Klincksieck, 2000), 95-108.

PARENT-CHARON, Annie, Les Métiers du livre à Paris au XVI' siècle (15351560) (Genève : Droz, 1974), « Le public du livre », 27-32.

PITTION, Jean-Paul, Le Livre à la Renaissance : introduction à la bibliographie historique et matérielle, coll. "Nugæ humanisticæ " (Turnhout : Brepols, 2013), «Le livre, objet de commerce » et « Le livre, objet culturel », 235306 et 307-355.

PUPPO, Dario Del, «All the World is a Book : Italian Renaissance Printing in a Global Perspective», Textual Culture 6.2 (2011) : 1-22.

RÉACH-NGÔ, Anne, éd., Créations d'atelier : l'éditeur et la fabrique de l'œuvre à la Renaissance, coll. "Études et essais sur la Renaissance »(Paris : Classiques Garnier, 2014), 395 p.

ROUDAUT, François, "Classements et bibliothèques à la Renaissance : quelques éléments ", Babel. Littératures plurielles 6 (2002) : 151-168.

SHARPE, Kevin et Steven N. ZWICKER, "Discovering the Renaissance Reader ", in Reading, Society and Politics in Early Modern England, éd. Kevin Sharpe et Steven N. Zwicker (Cambridge : Cambridge University Press, 2003), 1-40.

SIMONIN, Michel, «Des projets littéraires et de leurs réalisations éditoriales à la Renaissance ", Cahiers de l'Association internationale des études françaises 51 (1999) : 183-203.

SUHR, Carla, Publishing for the Masses. Early Modern English Witchcraft Pamphlets (Helsinki : Société Néophilologique, 2011), 318 p. 
TENGER, Zeynep et Paul TROLANDER, « From Print versus Manuscript to Sociable Authorship and Mixed Media : A Review of Trends in the Scholarship of Early Modern Publication ", Literature Compass 7.11 (2010) : 1035-1048.

VARRY, Dominique, éd., 50 ans d'histoire du livre, 1958-2008, coll. " Papiers » (Villeurbanne : Presses de l'ENSSIB, 2014), 223 p.

VARRY, Dominique, "Les ventes publiques de livres à Lyon aux XVII et XVIII siècles et leurs catalogues ", in Les Ventes de livres et leurs catalogues, $X V I I^{e}-X X^{e}$ siècles, éd. Annie Charon et Elisabeth Parinet (Paris : École des Chartes, 2000), 29-48.

WAKELIN, Daniel, Humanism, Reading, \& English Literature 1430-1530 (Oxford, New York : Oxford University Press, 2007), "Print and the Reproduction of Humanist Readers », 126-159.

WALSBY, Malcolm et Graeme KEMP, éd., The Book Triumphant. Print in Transition in the Sixteenth and Seventeenth Centuries, coll. " Library of the Written Word» (Leyde, Boston : Brill, 2011), 378 p.

WALTER, Melissa, "Constructing Readers and Reading Communities : Marguerite de Navarre's Heptaméron 32 in England ", Renaissance and Reformation / Renaissance et Réforme 27.1 (2003) : 35-59.

WARNER, Lyndan, The Ideas of Man and Woman in Renaissance France : Print, Rhetoric, and Law, coll. «Women and Gender in the Early Modern World » (Farnham, Burlington : Ashgate, 2011), "Booksellers and the Market to the 1550s », 25-50. 